Review Article

\title{
Mitochondrial Function and Mitophagy in the Elderly: Effects of Exercise
}

\author{
Osvaldo C. Moreira, ${ }^{1,2}$ Brisamar Estébanez, ${ }^{1}$ Susana Martínez-Florez, ${ }^{1}$ José A. de Paz, \\ María J. Cuevas, ${ }^{1}$ and Javier González-Gallego ${ }^{1}$ \\ ${ }^{1}$ Institute of Biomedicine (IBIOMED), University of León, León, Spain \\ ${ }^{2}$ Institute of Biological Sciences and Health, Federal University of Viçosa-Campus Florestal, Florestal, MG, Brazil
}

Correspondence should be addressed to María J. Cuevas; mjcueg@unileon.es and Javier González-Gallego; jgonga@unileon.es

Received 16 March 2017; Revised 4 June 2017; Accepted 6 July 2017; Published 16 August 2017

Academic Editor: Orlando Laitano

Copyright (C) 2017 Osvaldo C. Moreira et al. This is an open access article distributed under the Creative Commons Attribution License, which permits unrestricted use, distribution, and reproduction in any medium, provided the original work is properly cited.

\begin{abstract}
Aging is a natural, multifactorial and multiorganic phenomenon wherein there are gradual physiological and pathological changes over time. Aging has been associated with a decrease of autophagy capacity and mitochondrial functions, such as biogenesis, dynamics, and mitophagy. These processes are essential for the maintenance of mitochondrial structural integrity and, therefore, for cell life, since mitochondrial dysfunction leads to an impairment of energy metabolism and increased production of reactive oxygen species, which consequently trigger mechanisms of cellular senescence and apoptotic cell death. Moreover, reduced mitochondrial function can contribute to age-associated disease phenotypes in model organisms and humans. Literature data show beneficial effects of exercise on the impairment of mitochondrial biogenesis and dynamics and on the decrease in the mitophagic capacity associated to aging. Thus, exercise could have effects on the major cell signaling pathways that are involved in the mitochondria quality and quantity control in the elderly. Although it is known that several exercise protocols are able to modify the activity and turnover of mitochondria, further studies are necessary in order to better identify the mechanisms of interaction between mitochondrial functions, aging, and physical activity, as well as to analyze possible factors influencing these processes.
\end{abstract}

\section{Introduction}

Aging is a natural and inevitable process characterized by a progressive decline of individuals both physically and mentally. With age, there is a gradual accumulation of dysfunctional components that results in the deterioration of different biological functions, which finally increases the risk of death [1]. Autophagy, a catabolic mechanism, involves the degradation of damaged cellular and molecular components through the formation of a double membrane structure known as autophagosome, which fuses with the lysosome to form the autophagolysosome, surrounding the structures that will be degraded [2]. Autophagy can be classified according to its selective organelle. Thus, when the target is the mitochondria, it is called mitophagy; ribophagy for the ribosomes; peroxyphagy for the peroxisomes; or reticulophagy for the endoplasmic reticulum, among others [3]. Moreover, it is important to highlight that, although appropriate levels of autophagy induction are required to extend lifespan, a malfunctioning of autophagy occurs in many old organs and tissues [4].

Aging particularly affects mitochondrial homeostasis [5], and it is clear that mitochondria could contribute to aging due to its key role in the complex balance of cellular processes [6]. Age-related changes also affect mitochondrial membrane potential $(\Delta \Psi \mathrm{m})[7]$, inducing the opening of permeability transition pores in the mitochondrial membrane, which leads to mitochondrial depolarization, that is, a decrease in the $\Delta \Psi \mathrm{m}$ to $-100 \mathrm{mV}$ [8]. Moreover, the age-dependent decline of mitophagy hinders the elimination of dysfunctional or damaged mitochondria and alters mitochondrial biogenesis, leading to a progressive accumulation of mitochondria. These effects result in the deterioration of cellular function [9]. Therefore, physiological aging has been associated with 
decreased mitophagic processes but also with impaired mitochondrial functions.

It is important to highlight that many interventions leading to health improvement and extension in lifespan, such as calorie restriction, or treatment with rapamycin, spermidine, metformin, or the antioxidant resveratrol, also induce an activation of the autophagic/mitophagic machinery [4]. In addition, regular physical activity has demonstrated benefits on adults' health and has been also identified as an inducer of autophagy in vivo $[2,10]$. In the same line, recent evidences have demonstrated that exercise is also able to affect the activity and turnover of mitochondria by increasing biogenesis and mitophagy [11].

In this review, we summarize the role that mitochondria, including its biogenesis, dynamics, and mitophagy, play in the aging process, and how modulation of those functions contributes to the mitochondrial adaptations to physical exercise in the elderly.

\section{Mitochondria and Aging: Functions and Importance}

Mitochondria are the powerhouses of the cell, generating a large part of cellular ATP. Moreover, mitochondria are involved in calcium metabolism, contribute to the formation of intracellular reactive oxygen species (ROS), and play a leading role in the initiation of apoptosis, being therefore the key in maintaining cellular homeostasis and acting as important signaling organelles in different tissues [12]. The size and total number of mitochondria depend on the metabolic needs, the state of differentiation, and the different physiological conditions of the cell [13].

To maintain a healthy status, mitochondria regulate their biogenesis and engage in several dynamic behaviors. The key elements are depicted in Figure 1. Mitochondrial biogenesis is defined as the coordinated regulation between nuclear gene expression, protein import and transcription of mitochondrial DNA (mtDNA) [14]. This process is regulated by a several transcription factors, such as mitochondrial transcription factor A (TFAM) and the peroxisome proliferator-activated receptor gamma coactivator 1 -alpha (PGC-1 $\alpha$ ), as well as at a posttranscriptional level [15]. PGC- $1 \alpha$ is also involved in other functions, such as control of mitochondrial genome copy number, regulation of mitochondrial dynamics and modulation of oxidative phosphorylation [16]. Moreover, these organelles are highly dynamic and undergo fusion (the joining of two organelles into one) and fission (the division of a single organelle into two) [17]. Fusion process involves three GTPases; mitofusin (Mfn) 1 and Mfn2, which mediate outer mitochondrial membrane (OMM) fusion, and optic atrophy protein (OPA) 1, which regulates inner mitochondrial membrane (IMM) fusion. On the other hand, fission process is mediated by GTPase dynamin-related protein 1 (Drp1) and generates a polarized and a depolarized mitochondrion [18]. Mitochondria also undergo other dynamic behaviors, such as transport (directed movement within a cell) and degradation (targeted destruction via the mitophagic pathway). All these processes are essential for maintaining a healthy mitochondrial population [17].
The maintenance of mitochondrial structural integrity, biogenesis and function is essential to the cells, since mitochondrial dysfunction can induce disturbances in energy metabolism, increase ROS production and, consequently, trigger mechanisms of apoptotic cell death [12]. Moreover, during the last decades, multiple lines of evidence in model organisms and humans have demonstrated that impaired mitochondrial function can contribute to the aging process, as well as age-associated diseases $[19,20]$. In fact, it has been shown that decreased mitochondrial performance is a hallmark of aging possibly due to the central role of mitochondria in metabolism and cellular function. Thus, the potential toxicity of mitochondrial ROS (mtROS), originating from mitochondrial respiratory chain, led to the formulation of the oxidative stress theory of aging, which suggested that the accumulation of oxidative damage to macromolecules is an important point in the aging process [21]. Mitochondrial DNA has two characteristics that make it a key target of mtROS: on the one hand, its proximity to the respiratory chain and, on the other, the lack of protective histones. Damaged mitochondrial DNA alters the respiratory chain, increasing the free radical generation and triggering a vicious cycle. These changes result in organic dysfunction and aging phenotype [22]. Recently, however, in contrast to the original theory favoring oxidative damage as a cause for mtDNA mutations and corresponding declines in mitochondrial function, there are strong data arguing that most mammalian mtDNA mutations originate as replication errors made by the mitochondrial DNA polymerase $[6,19]$.

Additionally, the protein quality control, or proteostasis, plays an important role in age-related decline driving to the accumulation of misfolded and unfolded proteins and to the mitochondrial dysfunction. To solve this problem, and promote cell survival and organism longevity, cells activate a transcriptional response known as mitochondrial unfolded protein response (mtUPR) [23]. In response to the accumulation of unfolded proteins or dysfunctional oxidative phosphorylation system (OXPHOS), cells activate the mtUPR to recover mitochondrial function through the stabilization of mitochondrial protein-folding environment and upregulation of a cytosolic source of ATP production [24, 25]. In addition, to compensate for the OXPHOS activity reduction, mtUPR can induce mitochondrial biogenesis [25]. Finally, if despite mtUPR activation the cell cannot maintain a $\Delta \Psi \mathrm{m}$, the mitochondria are directed to the mitophagy pathway [26].

In aging, skeletal muscle mass decreases from midlife onwards. In addition, mitochondrial functional capacity and mitochondrial density are also reduced [27-29]. However, it is unclear whether these changes are a direct consequence of aging per se, or are due to inactivity. Indeed, a significant improvement in mitochondrial function and an increase in PGC- $1 \alpha$ expression have been observed in old trained subjects [30, 31]. The expression of deleterious mtDNA mutations within cells seems to be also controlled by the balance between fusion and mitochondrial fission. While fusion may allow the "dilution" of a mutant mtDNA species in a set of wild-type molecules, reducing the functional effect, fission may lead to the removal of aberrant 


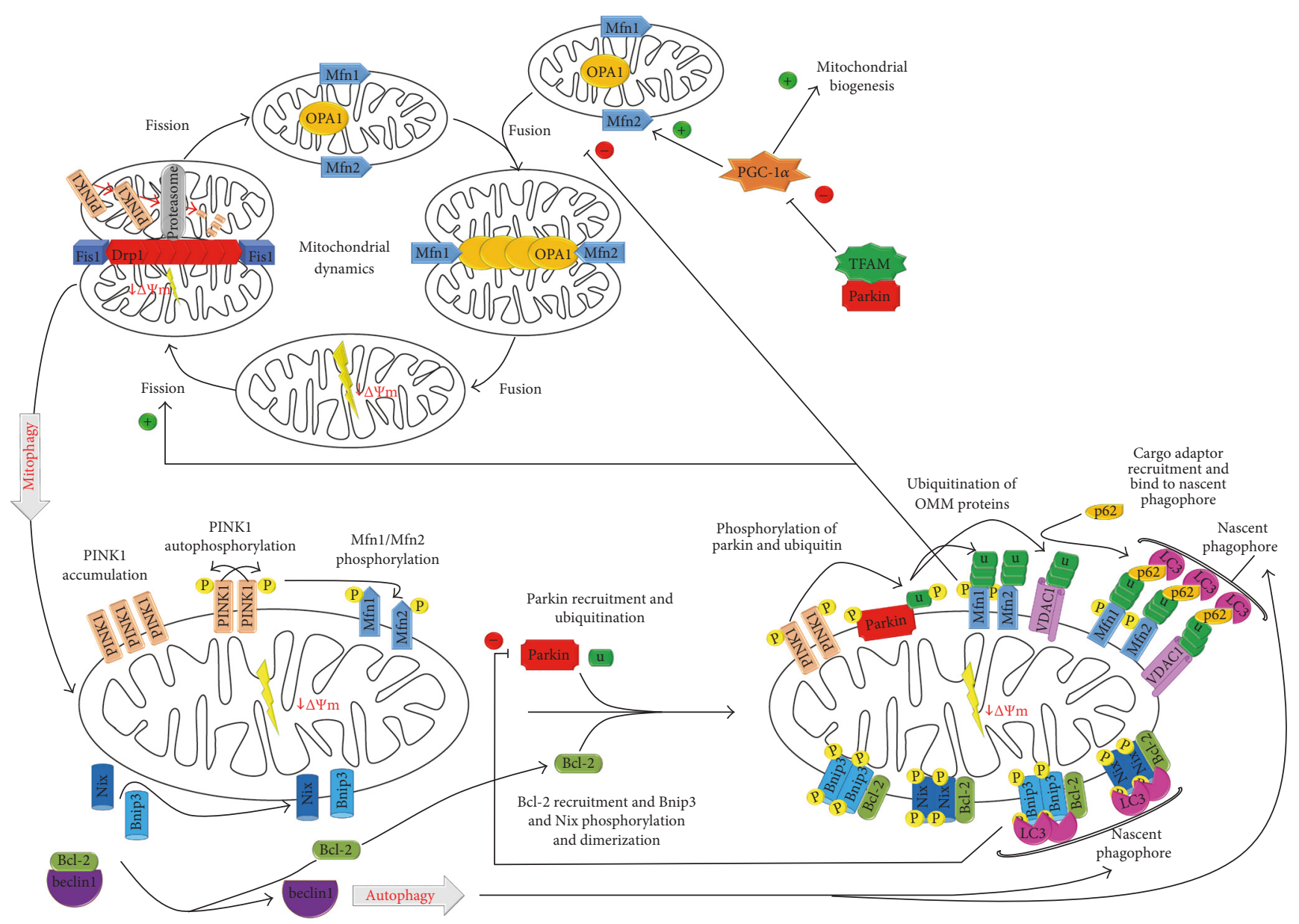

FIGURE 1: Mitochondrial biogenesis, dynamics, and mitophagy process. Machinery regulating mitochondrial morphology dynamics can regulate mitophagy initiation, so mitophagy is inhibited by fusion process, mediated via Mfn1/2 and OPA1, and promoted by fission process, mediated via Drp1. Fission process generates polarized mitochondria, which is driven to fusion process, and depolarized mitochondria, which is targeted by mitophagy. In polarized mitochondria, PINK1 is imported into IMM and degraded via proteasome. In depolarized mitochondria, PINK1 is accumulated in OMM. There, PINK1 recruits Parkin from the cytosol by Mfn1 and Mfn2 phosphorylation and phosphorylates ubiquitin and ubiquitin-like domain of Parkin. Then, Parkin ubiquitinates OMM proteins, such as VDAC1, which recruit the p62/SQSTM1 autophagy cargo adaptor. This receptor simultaneously binds to LC3 localized in the nascent phagophore. Furthermore, mitochondrial biogenesis can promote the fusion process, blocking mitophagy, through PGC-1 $\alpha$, which stimulates Mfn2 expression; and mitophagy can inhibit mitochondrial biogenesis via Parkin, whose association with TFAM inhibits the expression of PGC-1 $\alpha$. On the other hand, upon expression, Bnip3 and Nix bind Bcl-2, broking the beclin1/Bcl-2 interaction, so that beclin 1 can induce autophagy initiation. Moreover, Bnip3 and Nix are phosphorylated and form homodimers, which integrate in OMM and then bind to LC3. In both cases, the LC3 bond triggers mitochondria to autophagy.

mitochondria via selective mitophagy [6]. Thus, the polarized mitochondrion can be triggered to the fusion process and depolarized mitochondrion is targeted by mitophagy. In this way, mitophagy is promoted and inhibited by fission and fusion events, respectively [18]. Moreover, mitochondrial dynamics also regulates mtUPR and mitochondrial biogenesis, since Mfn2 overexpression causes increased mitochondrial membrane potential, glucose oxidation, and increased expression of nuclear-encoded subunit OXPHOS complexes I, IV, and V, while Mfn2 depletion represses the expression of complexes I, II, III, and V (a subunit). In addition, PGC- $1 \alpha$ promotes Mfn2 expression [20]. Finally, the balance between biogenesis and degradation plays a central role in the regulation of overall mitochondrial mass. Therefore, the mitophagic machinery seems to be the key in the clearance of the excess of mitochondria or of those old or defective mitochondria [17]. Thus, these mechanisms of cell destruction are activated in order to preserve the health and good working of tissues, since the increase of oxidative stress in tissues could cause DNA damage and mutations that accelerate the aging of the tissue and decrease its longevity [11].

\section{Mitophagy Mechanisms}

Since mitochondria are involved in both adaptive metabolism and survival in response to cellular stress, it is necessary to maintain good mitochondrial functioning through a tight mitochondrial quality control [32]. Recently, mitophagy has gained importance because the damage accumulated in the 
mitochondria may result in a large number of cell consequences. This process of dysfunctional mitochondria removal occurs by two major pathways, damage-induced mitophagy and developmental-induced mitophagy [13]. Mitophagy not only clears dysfunctional mitochondria but also participates in adaptive response to nutrient deprivation, hypoxia or developmental signals, promoting a reduction in the overall mitochondrial mass [32].

Damage-induced mitophagy has the function of removing damaged/defective mitochondria. It is driven by two main proteins: PTEN-induced putative kinase (PINK), which can sense mitochondrial polarization state, and the E3 ubiquitin protein ligase (Parkin) (Figure 1). In normal conditions, where mitochondria are polarized, PINK1 is maintained in low basal levels. For that, PINK1 is imported into IMM and processed by mitochondrial peptidases, such as the protease presenilin-associated rhomboid-like protein (PARL) [16]. However, in damaged/defective mitochondria, which are depolarized, this process is inactivated since $\Delta \Psi \mathrm{m}$ is insufficient to transfer PINK1 to IMM, accumulating at the OMM $[33,34]$. There, some mitochondrial proteins are phosphorylated by PINK1, which results in the recruitment of autophagy cargo adaptors that bind to the autophagosome through light chain 3 protein (LC3) and, subsequently, to the lysosome. Thereby, mitochondria are degraded by autophagolysosomes [32]. To amplify this signal, PINK1 recruits Parkin from the cytosol by Mnf1 and Mnf2 phosphorylation. In addition, PINK1 phosphorylates ubiquitin and ubiquitin-like (Ubl) domain of Parkin, which activates Parkin E3 ligase activity and facilitates its recruitment to OMM [18].

Once on the OMM, Parkin ubiquitinates OMM proteins, in addition to ubiquitinating itself [35]. First, Parkin can trigger the ubiquitination of Mfn1 and Mfn2, preventing the fusion of the mitochondria and working as a mechanism of isolation when these organelles are damaged, for subsequent sequestration and degradation by selective autophagy [36]. Then, Parkin ubiquitinates OMM proteins, such as voltagedependent anion channel (VDAC) 1, generating polyubiquitin chains via Lys27, so that recruits LC3-interacting region- (LIR-) containing autophagy receptors, such as sequestosome (p62/SQSTM), which simultaneously binds to LC3, pointing to the mitochondria that should be eliminated $[13,18]$. Additionally, Parkin regulates mitochondrial biogenesis through its association with TFAM, which inhibits the expression of PGC- $1 \alpha$ as well as its target gen, the nuclear respiratory factor (NRF) 1 [15].

Developmental process-induced mitophagy has the function of programmatic elimination of excessive mitochondrial population [13]. This type of mitophagy is driven by two main proapoptotic proteins, a member of the B-cell lymphoma 2 family (Bcl-2), adenovirus E1B $19 \mathrm{kDa}$-interacting protein (Bnip) 3 and Nix (Nip3-like protein X or Bnip3L), which induce mitophagy by means of several potential mechanisms [37]. However, mitophagy mediated by Bnip3/Nix is different from the PINK1/Parkin pathway, since PINK1 and Parkin proteins cannot bind directly to autophagosome receptors, while Bnip3 and Nix directly bond the autophagy machinery components (Figure 1) [38].
Bnip3 can induce autophagy through several mechanisms, including mitochondrial depolarization, mitochondrial permeability transition pore (MPTP) aperture, or interference in fission-fusion machinery [39]. Phosphorylation regulates mitophagy activity of Bnip3. This phosphorylation promotes binding to LC3II and Golgi-associated ATPase enhancer of $16 \mathrm{kDa}$ (GATE-16), and phosphorylation of serine residue 24 increases the affinity for them. On the other hand, phosphorylation at residues at its C-terminus, adjacent to the transmembrane domain, decreases the mitochondrial damage generated by Bnip3 and inhibits cell death but does not block autophagy/mitophagy. Additionally, Bnip3 increases the location of Drp1 to mitochondria [40].

Nix is already required for programmed mitophagy during maturation of reticulocytes, which includes the removal of membrane organelles, such as mitochondria, through a process related to autophagy, with the main difference that the content of the autophagy vacuole is not recycled but eliminated by exocytosis [39]. It is possible that Nix induces mitophagy by its interaction with LC3. LC3 interacts with gamma-aminobutyric acid receptor-associated protein (GABARAP), to form the LC3/GABARAP complex, and mediates mobilization of autophagosome to mitochondria to be eliminated $[18,36]$. In addition, Nix can also interact with protein encoded by the BECN1 gene (beclin1)/Bcl-2 complex to release beclin1, thus freeing beclin1 to induce autophagy [13, 37].

Other phospho-regulated OMM-localized mitophagy receptors are FUN14 domain containing (FUNDC) 1 and Bcl-2-like protein 13 (Bcl2-L-13)/Bcl-Rambo, which promote mitophagy through directly binding to components of the autophagy machinery [38]. Furthermore, OMMlocalized lipids, such as ceramide, a sphingolipid, or cardiolipin, a dimeric phospholipid, can also recruit mitophagic machinery binding directly to LC3 $[18,41]$.

\section{Effects of Aging on Mitochondrial Function and Mitophagy}

A proper working maintenance of both mitochondrial quantity and quality is strictly related to the conservation of an adequate concentration of several proteins, such as PGC-1 $\alpha$, TFAM, OPA1, Drp1, Mfn1, Mfn2, mitochondrial fission (Fis) 1 protein, PINK1, Parkin, VDAC1, Bnip3, and Nix, which are involved in mitochondrial biogenesis, dynamics and mitophagy process. However, organic alterations, characteristic of aging, can modify the concentration of these proteins, destabilizing their functions (Table 1).

Some studies that relate autophagy and/or mitochondrial function to the aging process show results that may be different $[10,42-44]$. Specifically, regarding proteins involved in biogenesis, most studies found a decrease in mRNA and protein expression of PGC- $1 \alpha$ [45-53]. These data indicate that, during aging, the reduced mitochondrial biogenesis may be due to the lack of response of PGC- $1 \alpha$ to different stimuli. In fact, aged rats behave as PGC- $1 \alpha$ knockout mice [54]. On the contrary, TFAM, another transcription factor that regulates mitochondrial synthesis, seems to be increased in 
TABLE 1: Effect of aging on mitochondrial proteins.

\begin{tabular}{|c|c|c|c|c|}
\hline Reference & Tissue & Subject & Comparative & Results \\
\hline$[58]$ & Muscle & Humans & $\begin{array}{c}\text { Sedentary and active youths and } \\
\text { elderly }\end{array}$ & NS (mRNA): PGC-1 $\alpha$; TFAM; Fis1; Mfn1 \\
\hline [45] & Carotid arteries & Mouse & $\begin{array}{l}\text { Sedentary and active youths and } \\
\text { elderly }\end{array}$ & $\begin{array}{c}\downarrow: \text { PGC- } 1 \alpha ; \text { Mfn } 2 \\
\uparrow: \text { Fis } 1\end{array}$ \\
\hline [46] & Muscle & Humans & Youths/elderly & $\begin{array}{c}\downarrow: \text { PGC- } 1 \alpha \text {; OPA1 } \\
\text { NS: Mfn2; Drp1; Fis1 }\end{array}$ \\
\hline [47] & Muscle & Rats & Youths/elderly & $\downarrow$ (mRNA and protein): PGC- $1 \alpha$; TFAM \\
\hline [73] & Muscle & Mouse & Youths/elderly & $\uparrow:$ Nix; LC3 \\
\hline [48] & Muscle & Rats & Youths/elderly & $\begin{array}{c}\downarrow: \text { PGC- } 1 \alpha \\
\uparrow: \text { TFAM; Fis } 1 ; \text { Mfn } 1\end{array}$ \\
\hline$[30]$ & Muscle & Humans & Youths/elderly & NS: PGC- $1 \alpha$; Mfn1; Mfn2; Fis1 \\
\hline [49] & Muscle & Humans & Youths/elderly & NS: PGC- $1 \alpha$; TFAM \\
\hline [68] & Muscle & Humans & Youths/elderly & $\begin{array}{l}\text { NS (mRNA): PGC-1 } \alpha \text {; TFAM; beclin1; Bnip3 } \\
\text { NS: Parkin; PINK1; VDAC1 }\end{array}$ \\
\hline$[50]$ & Muscle & Humans & $\begin{array}{l}\text { Youths and active and } \\
\text { sedentary elderly }\end{array}$ & $\downarrow:$ PGC- $1 \alpha$ \\
\hline [72] & Muscle & Humans & $\begin{array}{l}\text { Youths and active and } \\
\text { sedentary elderly }\end{array}$ & $\begin{array}{c}\uparrow: \text { PGC-1 } \alpha \text {; beclin1 (elderly versus youths); Bnip3 } \\
\text { (sedentary elderly versus youths) } \\
\text { NS: Bnip3 (active elderly versus youths) }\end{array}$ \\
\hline [71] & Muscle & Humans & Youths/elderly & $\begin{array}{c}\text { NS: VDAC1 } \\
\downarrow \text { : beclin1; Fis1 }\end{array}$ \\
\hline$[43]$ & Muscle & Mouse & Youths/elderly & $\uparrow:$ Mfn1; Mfn2 \\
\hline [67] & Heart & Rats & $\begin{array}{l}\text { Youths, old adult, } \\
\text { and senescent }\end{array}$ & $\begin{array}{c}\text { NS: PGC-1 } \alpha \text {; TFAM; OPA1; Drp1; LC3II } \\
\downarrow \text { : Bnip3; Drp1; Fis1 } \\
\uparrow \text { : OPA1 (old adults versus youths); Mfn2; LC3I } \\
\text { NS: beclin1; LC3II }\end{array}$ \\
\hline [59] & $\begin{array}{l}\text { Gastrocnemius }(\mathrm{G}) \\
\text { and triceps }(\mathrm{T}) \\
\text { muscle }\end{array}$ & Rats & Youths/elderly & $\begin{array}{l}\downarrow \text { (G): Drp1; Fis1; beclin1; LC3II; (T): PINK1; VDAC1 } \\
\uparrow(G) \text { : OPA1; Mfn1; VDAC1; (T): OPA1; Mfn1; Drp1; } \\
\text { Fis1 } \\
\text { NS: (G): PINK1; (T) beclin1; LC3II }\end{array}$ \\
\hline [51] & Muscle & Rats & Youths/elderly & $\begin{array}{c}\downarrow: \text { PGC }-1 \alpha \\
\uparrow: \text { TFAM }\end{array}$ \\
\hline [55] & $\begin{array}{l}\text { Cerebellum, heart, } \\
\text { kidney, and liver }\end{array}$ & Rats & Youths/elderly & $\begin{array}{c}\uparrow: \text { TFAM } \\
\text { NS (heart): TFAM }\end{array}$ \\
\hline [66] & Muscle & Humans & $\begin{array}{l}\text { Youths, middle-aged, } \\
\text { and elderly }\end{array}$ & NS: OPA1; Mfn2; Fis1; Drp1; Bnip3; beclin1; LC3II/I \\
\hline [60] & Muscle & Mouse & Youths/elderly & $\begin{array}{c}\uparrow: \text { Mfn2/Drp1 } \\
\text { NS: OPA; Drp1; Mfn1; Mfn2 }\end{array}$ \\
\hline [44] & Muscle & Rats & Youths/elderly & $\begin{array}{l}\text { NS: Drp1; beclin1; LC3II } \\
\uparrow: \text { Parkin; Fis1; OPA1; Mfn2 }\end{array}$ \\
\hline [56] & Brain & Rats & Youths/elderly & $\uparrow:$ TFAM \\
\hline [52] & Liver & Rats & $\begin{array}{l}\text { Middle-aged, old }(18 \mathrm{~m}) \\
\text { and very old }(28 \mathrm{~m})\end{array}$ & $\begin{array}{l}\downarrow: \text { TFAM; PGC- } 1 \alpha \\
\text { NS: VDAC }\end{array}$ \\
\hline [57] & $\begin{array}{l}\text { Brain, muscle, and } \\
\text { liver }\end{array}$ & Rats & $\begin{array}{l}\text { Middle-aged, old }(18 \mathrm{~m}) \\
\text { and very old }(28 \mathrm{~m})\end{array}$ & $\begin{array}{c}\uparrow \text { (brain): TFAM } \\
\downarrow \text { (muscle and liver): TFAM } \\
\uparrow: \text { Bnip3; LC3II; LC3II/I }\end{array}$ \\
\hline [64] & Muscle & Mouse & Youths/elderly & $\begin{array}{l}\downarrow: \text { Mfn2 } \\
\text { NS: LC3I }\end{array}$ \\
\hline
\end{tabular}


TABle 1: Continued.

\begin{tabular}{|c|c|c|c|c|}
\hline Reference & Tissue & Subject & Comparative & Results \\
\hline [61] & Brain & Mouse & $\begin{array}{l}\text { Youth, middle-aged, } \\
\text { and elderly }\end{array}$ & $\begin{array}{c}\downarrow: \text { TFAM; Drp } 1 ; \text { Mfn2 } \\
\uparrow: \text { OPA1; Mfn } 1\end{array}$ \\
\hline$[53]$ & Heart & Humans & Youths/elderly & $\downarrow:$ PGC- $1 \alpha$ \\
\hline [75] & Muscle & Humans & Youths/elderly & $\begin{array}{l}\downarrow \text { : LC3II/I } \\
\text { NS: beclin1 }\end{array}$ \\
\hline [10] & PBMCs & Humans & Youths/elderly & $\downarrow$ : LC3II/I; beclin 1 \\
\hline
\end{tabular}

the muscle and brain from old rats and decreased in the liver or in the muscle from very old rats [48, 51, 55-57]. In humans, TFAM decreases or does not change significantly in the skeletal muscle $[49,58]$. Those results seem to indicate that several tissue-specific mitochondrial changes associated with aging might be due to a fine modulation of TFAM binding to mtDNA.

OPA1 is located on the IMM and regulates mitochondrial fusion and ridge structure. In addition, OPA1 can control mitophagy by promoting the stabilization of mitochondrial ridges, which, in turn, act against mitochondrial dysfunction and excessive production of ROS. Thus, a higher expression of this protein indicates an increase in mitochondrial fusion and a decrease in mitophagy [33]. Most research seems to indicate an increase of this protein in the muscle and brain of aged rodents [44, 59-61]. OPA1 acts as a mitochondrial fusion factor, perhaps by interacting with mitofusins or other outer membrane fusion factors $[62,63]$. In this line, Mfn1 and Mfn2 also increased in mouse and rat muscles during senescence [43, 44, 48, 59-61]. However, other studies point to an absence of changes or even decreases in these proteins responsible for mitochondrial fusion $[30,45,46,58,60,64-66]$. On the other hand, and as previously mentioned, mitophagy is promoted by fission [18]. Most authors have shown a decrease in Drp1 levels in the heart, brain, and muscle from old rodents [59-61, 67]. Moreover, the ratio between Mfn2 and Drp1, an index of the balance between fusion and fission processes, was significantly increased in atrophied skeletal muscle of aged mice [60]. These results reinforce the possibility that OPA1 might act as an inhibitor of mitochondrial fission [64]. However, results about the role of Fis1 protein in the aging process are not conclusive [30, 44, 45, 48, 58, 59, 65-67].

PINK1 expression seems to be diminished in hepatocytes and liver tissue from mouse over 18 months of life [65], but in samples from human and rat muscles, no significant changes associated with age have been found [59, 68]. Other authors have reported an increase in muscle Parkin levels of old rats, which might be a further consequence of lipofuscin accumulation within lysosomes, resulting in a buildup of cellular debris [44]. Moreover, the dysregulation of mitochondrial dynamics with aging leads to the accumulation of unhealthy mitochondria, which could result in the recruitment of Parkin in order to control mitochondrial quantity and quality [69].

VDAC1 determines the permeability and conductance of the outer membrane and plays an important role as a modulator of permeability of membrane pores [33]. It has been demonstrated that the level of VDAC1 is significantly decreased in mitochondria isolated from the brain of old rats [70]. The lower protein content might be related to the changes in the mitochondrial susceptibility to $\mathrm{Ca}^{2+}$ overloading and for the permeability of membrane pore facilitation found with aging [70]. The same result was observed in triceps muscle from old rats [59]. However, other studies note that, apparently, aging does not decrease the expression of this protein in human muscles when young and old are compared [68, 71].

Regarding the effect of aging on the expression of Bnip3, literature does not seem to be very conclusive. Some studies have been found that this protein may increase in the muscles of sedentary elderly $[64,72]$. A possible explanation may be that Bnip3 induction compensates for the loss of mitochondrial autophagy and minimizes mitochondrial damage [64]. Conversely, Bnip3 was decreased in the heart of old rats [67] whereas in the muscle of aged humans it remained unchanged $[66,68]$. The effects of aging on Nix, and subsequently on autophagy and mitochondrial clearance, in the skeletal muscles are scarcely known. It has been reported that the expression of Nix increases in the skeletal muscles of old mice [73]. This observation, while consistent with research that shows an increase in autophagy markers in aged muscles [44], contrasts with others authors that have reported agingassociated decline in mitophagy $[9,74]$. Perhaps, the explanation for this discrepancy may be the extreme ages (3-5 months old versus 22-24 months old) of mice used in the study by Ko et al. [73]. Taking into account these data, it is possible to infer that the increase of both Bnip3 and Nix, associated with age, may indicate a failure in the mechanism of mitophagy. Possibly, Bnip3 and Nix accumulation could hinder these proteins to perform their function in the mitophagic process.

The autophagy markers beclin1 and LC3 (LC3II or LC3II/I) also seem to be altered in the aging process. Thus, beclin1 levels have been reported to decrease in human and mouse muscles $[43,66]$ and in peripheral blood mononuclear cells (PBMCs) from old subjects [10]. However, other studies in human and rat muscles did not show any change $[44,68,75]$. These differences could be due to the tissue type, as observed in gastrocnemius and triceps rat muscles [59]. Several studies have shown that LC3II or LC3II/ LC3I increased in mouse aged muscle [64], while other studies have described no changes in human and rat aged muscle $[44,66]$. On the other hand, previous results from our research group showed that the LC3II/I ratio was markedly lower in mononuclear cells from old subjects when 
compared to a young group [10]. A similar phenomenon has been also observed by others when young and older adult skeletal muscles were studied [75].

Overall, it has been reported that aging is associated with a decline in the mitochondrial function, in the accumulation of abnormal mitochondria and in the mitophagic capacity of the organism. However, studies analyzed do not exhibit a consensus in relation to most proteins involved. It is up to future research to determine more precisely the mechanisms of interaction between aging and mitochondrial functions, as well as to analyze possible factors that could influence this process, such as sex, nutritional pattern or habitual level of physical activity.

\section{Effects of Exercise on Mitochondrial Biogenesis, Dynamics, and Mitophagy in Aging}

Physical exercise has been proposed as a nondrug treatment against different diseases for people of all ages [76]. In addition, it is suggested that regular exercise could promote an increase in mitophagy capacity [14] and produce effects on the mitochondrial life cycle (Table 2).

Theoretically, physical exercise could also have effects on the major signaling pathways that are involved in the quality and quantity control of mitochondria during the aging process, such as mitophagy [77]. Mitochondria produce ROS that can act as signaling molecules, inducing a survival response or causing damage to cellular components. However, contraction of the skeletal muscle during physical exercise can activate a mitochondrial response that improves the quality of mitochondria in different ways: (1) increasing biogenesis; (2) enhancing the expression and action of the proteins involved in the mitochondrial dynamics, as OPA1; (3) raising mitochondrial turnover by the action of mitophagy proteins, such as PINK1, Parkin, Nix and Bnip3; and (4) increasing the quality control of mitochondria through the degradation of damaged or dysfunctional mitochondria [78].

In this regard, exercise would produce an immediate increase of mitochondrial activation involving a significant increase in the expression of the transcription factor PGC$1 \alpha$ and of nuclear genes encoding mitochondrial protein expression. All these modifications would result in a higher mitochondrial content, with better performance, such as raised oxygen consumption and ATP synthesis, reduced ROS production, and increased mitophagy capacity [31]. In fact, independent of the volume and intensity, regular endurance physical exercise induces the gene expression of PGC- $1 \alpha$ in the skeletal muscles from old human and rodents $[30,31,47,48,79]$. Even 7 days of electrostimulation [80] or a single bout of high intensity exercise, both endurance and resistance $[68,81,82]$, are able to increase this transcription factor, at mRNA level, in aged rat and human muscles [80]. Only in one study, based on a single bout of $45 \mathrm{~min}$ of moderate endurance exercise plus vigorous exercise until exhaustion, no changes in muscle PGC- $1 \alpha$ levels were observed [58]. In the same line, TFAM mRNA increased in the muscle after several weeks of aerobic exercise [31, 47] but no changes were detected in short trainings (3-6 weeks) $[48,83]$ or a single bout of exercise [58] carried out by old humans or rats.

OPA1 is another protein that may be unchanged in response to voluntary exercise $[46,84]$. However, it has been also demonstrated that 12 weeks of aerobic exercise increased OPA1 mRNA content in the skeletal muscle of old subjects [85]. The mechanism by which exercise could promote the increase of this protein is not established, but it seems to be related to the maintenance of higher levels of mitochondrial fusion and fission processes during exercise [86]. Although results are not conclusive, the increase showed in muscle Mfn1 mRNA during aging could be reversed by a single bout of running in humans [58] or 6 weeks of treadmill running training in rats [48]. However, other studies in human muscle demonstrated that Mfn1 and Mfn2 were unaltered in response to exercise $[45,46,83,84,87]$. On the other hand, although Mfn1 and Mfn2 were increased after 12 weeks of cycling [30], a similar aerobic protocol, with the same duration and higher intensity, did not show an increase in the Mfn1 and Mfn2 mRNAs [85]. The role of Drp1 and Fis1 is also contradictory in response to different types of exercise in the elderly [ $30,45,46,48,58,83,85,87]$. This fact was also described in the previous section when only the effect of the age was evaluated.

In relation to the proteins involved in mitophagy, it is possible to consider that a decrease in the bioavailability of PINK1 and Parkin1 may indicate a higher degree of mitochondrial dysfunction during aging [87]. Thus, the increase of these proteins, caused by exercise, may be an attempt to promote the control of mitochondrial quality through the action of the mitophagy machinery [69]. However, there is no general consensus on the effects of exercise during aging. Studies that evaluated PINK1 found that this protein may not suffer alterations in human muscles [68, 85, 87], suggesting that this pathway is not stimulated by exercise. On the other hand, muscle Parkin protein could remain unchanged [68, 85] or increase at the level of mRNA [87] in response to exercise practice in the elderly. It is important to highlight that, in those studies where no significant changes were observed in PINK1 or Parkin, there was a trend towards an increase in the expression of both proteins $[68,85]$. However, results from those studies must be taken with caution due to several reasons. For example, in the study by Ogborn et al. [68], it is remarkable that both, young and old, were grouped to demonstrate the effect of a single bout of resistance exercise over time, while Fealy et al. [85] recruited only obese old subjects (body mass index: $34.6 \mathrm{~kg} / \mathrm{m}^{2}$ ), who carried out an aerobic training during 12 weeks.

VDAC1, another Parkin-ubiquitinated protein, appears to exhibit no alterations in response to exercise. Thus, 20 sessions of aerobic cyclergometer training did not change muscle VDAC1 in old participants [71]. A single bout of resistance exercise also did not modify the protein content of VDAC1 [68]. So, further studies are needed to analyze the behavior of this protein in relation to the practice of physical exercise, to determine more precisely whether physical exercise can induce alterations in its expression. 
TABLE 2: Effect of exercise on mitochondrial proteins in aging.

\begin{tabular}{|c|c|c|c|c|c|}
\hline Reference & Tissue & Subject & Comparative & Training & Results \\
\hline$[58]$ & Muscle & Humans & $\begin{array}{l}\text { Sedentary and active } \\
\text { youths and elderly }\end{array}$ & $\begin{array}{l}\text { Single bout of treadmill running of } 45 \text { min at } \\
70-75 \% \mathrm{VO}_{2 \max } \text { plus exercise until exhaustion } \\
\text { at } 90 \% \mathrm{VO}_{2 \max }\end{array}$ & $\begin{array}{l}\downarrow \text { (mRNA): Fis1; Mfn1 } \\
\text { NS: PGC- } 1 \alpha \text {; TFAM }\end{array}$ \\
\hline$[31]$ & Muscle & Humans & $\begin{array}{l}\text { Sedentary elderly and } \\
\text { active elderly } \\
\text { Sedentary elderly } \\
+ \text { training }\end{array}$ & $\begin{array}{l}16 \text { weeks of aerobic exercise (bike, walk, } \\
\text { run, or row), } 3 \text { times/week at } 75-80 \% \text { HR }\end{array}$ & $\begin{array}{c}\uparrow \text { (mRNA): PGC- } 1 \alpha \text {, TFAM } \\
\text { (sedentary elderly }+ \text { training) } \\
\text { NS (mRNA): PGC- } 1 \alpha \text {, TFAM } \\
\text { (sedentary elderly versus active } \\
\text { elderly) }\end{array}$ \\
\hline$[81]$ & Muscle & Humans & $\begin{array}{l}\text { Trained elderly and } \\
\text { untrained elderly } \\
\text { Untrained elderly } \\
\text { + acute exercise }\end{array}$ & $\begin{array}{c}\text { Single bout of high intensity interval exercise } \\
\text { on a bicycle ergometer for } 20 \text { min at } 80 \% \\
\text { of peak power output }\end{array}$ & $\begin{array}{c}\uparrow \text { (mRNA): PGC- } 1 \alpha \\
\text { (untrained + acute } \\
\text { exercise) } \\
\uparrow: \text { PGC- } 1 \alpha \text { (trained versus } \\
\text { untrained elderly) } \\
\text { NS: PGC- } 1 \alpha \text { (untrained } \\
\text { + acute exercise) }\end{array}$ \\
\hline [87] & Muscle & Humans & $\begin{array}{l}\text { Sedentary elderly } \\
\text { Active elderly }\end{array}$ & Without exercise intervention & $\begin{array}{l}\uparrow \text { (mRNA): Bnip3; Drp1; } \\
\text { Parkin; beclin1 } \\
\text { NS (mRNA): Mfn2; } \\
\text { PINK1; LC3 } \\
\text { NS: beclin1 }\end{array}$ \\
\hline$[45]$ & $\begin{array}{l}\text { Carotid } \\
\text { arteries }\end{array}$ & Mouse & $\begin{array}{l}\text { Sedentary and active } \\
\text { youths and elderly }\end{array}$ & $\begin{array}{l}10 \text { weeks of voluntary aerobic exercise in a } \\
\text { wheel running }\end{array}$ & NS: PGC- $1 \alpha$; Fis1; Mfn2 \\
\hline [83] & Brain & Mouse & Elderly & $\begin{array}{l}3 \text { weeks of treadmill running for } 60 \mathrm{~min} \text { at } \\
15-19 \mathrm{~m} / \mathrm{min} \text { and } 10^{\circ} \text { incline }\end{array}$ & $\begin{array}{c}\text { NS (cortex): PGC-1 } \alpha \text {, } \\
\text { TFAM, Mfn2; LC3II } \\
\text { NS (striatum): PGC-1 } \alpha, \\
\text { TFAM, Drp1, Mfn2; LC3II } \\
\uparrow \text { (cortex): Drp1 }\end{array}$ \\
\hline$[82]$ & Muscle & Humans & Elderly & $\begin{array}{l}\text { Single bout of bicycle exercise at } 75 \% \mathrm{VO}_{2 \max } \\
\text { until exhaustion }\end{array}$ & $\begin{array}{l}\uparrow(\mathrm{mRNA}): \text { PGC- } 1 \alpha \\
\text { NS (mRNA): TFAM }\end{array}$ \\
\hline$[46]$ & Muscle & Humans & Youths/elderly & Without exercise intervention & $\begin{array}{l}\text { NS: PGC- } 1 \alpha \text {; TFAM; Mfn2; } \\
\text { OPA1; Drp1; Fis1 }\end{array}$ \\
\hline$[47]$ & Muscle & Rats & Youths/elderly & $\begin{array}{l}12 \text { weeks of treadmill running } 5 \text { times/week } \\
\text { for } 45 \mathrm{~min} \text { at } 17.5 \mathrm{~m} / \mathrm{min} \text { and } 10^{\circ} \text { incline }\end{array}$ & $\begin{array}{c}\uparrow: \text { PGC- } 1 \alpha \text {; TFAM } \\
\text { NS (mRNA): PGC- } 1 \alpha \text {; TFAM }\end{array}$ \\
\hline$[48]$ & Muscle & Rats & Youths/elderly & $\begin{array}{l}6 \text { weeks of treadmill running for } 60 \mathrm{~min} \text { at } \\
10-22 \mathrm{~m} / \mathrm{min} \text { and } 5-10 \% \text { incline }\end{array}$ & $\begin{array}{c}\uparrow: \text { PGC- } 1 \alpha \\
\downarrow: \text { Mfn } 1 \\
\text { NS: TFAM; Fis1 }\end{array}$ \\
\hline$[30]$ & Muscle & Humans & Youths/elderly & $\begin{array}{c}12 \text { weeks of cycling 3-4 times/week at } 60-80 \% \\
\text { HR reserve for } 20-45 \mathrm{~min}\end{array}$ & $\begin{array}{c}\uparrow: \text { PGC- } 1 \alpha \text {; OPA } 1 ; \text { Mfn } 1 ; \text { Mfn } 2 ; \\
\text { Fis } 1\end{array}$ \\
\hline [49] & Muscle & Humans & Youths/elderly & Without exercise intervention & $\uparrow:$ PGC- $1 \alpha$; TFAM \\
\hline$[80]$ & Muscle & Rats & Youths/elderly & $\begin{array}{l}7 \text { days of electroestimulation } 3 \mathrm{~h} / \text { day at } 10 \mathrm{~Hz} \\
\text { for } 0.1 \mathrm{~ms} \text { duration }\end{array}$ & $\uparrow:$ PGC- $1 \alpha$; TFAM \\
\hline$[68]$ & Muscle & Humans & Youths/elderly & $\begin{array}{c}\text { Single bout of resistance training (4 sets } \\
\text { of rept. at } 75 \% 1 \mathrm{RM} \text { with } 2 \text { min rest } \\
\text { between sets) }\end{array}$ & $\begin{array}{c}\uparrow(\mathrm{mRNA}): \text { PGC-1 } \alpha(3 \mathrm{~h}) ; \\
\text { TFAM }(24 \mathrm{~h}) ; \mathrm{LC} 3 \mathrm{II}(3 \mathrm{~h}) \\
\uparrow: \text { LC3II }(48 \mathrm{~h}) \\
\text { NS (mRNA): Nix; Bnip3 } \\
\text { NS: Parkin; PINK1; VDAC1; } \\
\text { beclin1 }\end{array}$ \\
\hline$[79]$ & Muscle & Mouse & Youths/elderly & $\begin{array}{c}6 \text { weeks of voluntary aerobic exercise in } \\
\text { wheels running }\end{array}$ & $\uparrow:$ PGC- $1 \alpha$ \\
\hline$[84]$ & Muscle & Mouse & Elderly & $\begin{array}{l}\text { Single bout of voluntary aerobic exercise } \\
\text { in wheels running for } 3 \mathrm{~h}\end{array}$ & NS: OPA1; Mfn2 \\
\hline
\end{tabular}


TABLE 2: Continued.

\begin{tabular}{|c|c|c|c|c|c|}
\hline Reference & Tissue & Subject & Comparative & Training & Results \\
\hline$[72]$ & Muscle & Humans & $\begin{array}{l}\text { Youths and active and } \\
\text { sedentary elderly }\end{array}$ & Without exercise intervention & $\begin{array}{c}\downarrow: \text { Bnip } 3 \\
\text { NS: PGC- } 1 \alpha \text {; beclin } 1\end{array}$ \\
\hline [85] & Muscle & Humans & Elderly & $\begin{array}{l}12 \text { weeks of aerobic exercise for } 60 \mathrm{~min} \\
(20 \text { min cycle ergometer and } 40 \mathrm{~min} \\
\text { treadmill walking) at } 80-85 \% \mathrm{HR}_{\max } \\
5 \text { days/week }\end{array}$ & $\begin{array}{c}\uparrow(\text { mRNA): OPA1; Drp1 } \\
\downarrow \text { : phosphorylated Drp1 } \\
\text { NS (mRNA): Mfn1; Mfn2; Fis1; } \\
\text { PINK1; Parkin }\end{array}$ \\
\hline [71] & Muscle & Humans & Youths/elderly & $\begin{array}{l}14 \text { days of immobilization and } 20 \text { sessions } \\
\text { of aerobic cycle ergometer training with } \\
48-58 \text { min at } 85 \% \mathrm{HR}_{\max }\end{array}$ & $\begin{array}{l}\uparrow \text { (immobilization }+ \text { training } \\
\text { versus immobilization): } \\
\text { VDAC1 } \\
\text { NS (immobilization + training } \\
\text { versus control): VDAC1 }\end{array}$ \\
\hline [89] & Muscle & Humans & Elderly & $\begin{array}{l}24 \text { weeks of combined exercise (walking }+ \\
\text { strength training + flexibility) } 2-3 \text { times/week } \\
\text { at moderate intensity }\end{array}$ & $\begin{array}{c}\text { NS (mRNA): Bnip3 } \\
\uparrow(\text { mRNA): PGC- } 1 \alpha \text {; TFAM; } \\
\text { LC3II } \\
\uparrow: \text { TFAM }\end{array}$ \\
\hline$[75]$ & Muscle & Humans & Youths/elderly & $\begin{array}{l}5 \text { days of bed rest and } 8 \text { weeks of high } \\
\text { intensity resistance exercise }\end{array}$ & $\begin{array}{c}\uparrow: \text { LC3II/I } \\
\text { NS: beclin1 }\end{array}$ \\
\hline$[10]$ & PBMCs & Humans & Elderly & 8 weeks of aerobic training & $\uparrow:$ LC3II/I, beclin 1 \\
\hline [2] & PBMCs & Humans & Elderly & 8 weeks of resistance training & $\begin{array}{c}\uparrow: \text { beclin } 1 \\
\text { NS: LC3II/I }\end{array}$ \\
\hline
\end{tabular}

Nix mRNA increases in the muscle sample taken from the vastus lateralis after ultraendurance exercise in human adults [88]. This protein exerts a direct regulation of autophagy, as an increase in its expression may indicate a higher formation of autophagosomes and greater mitophagy flux. In this way, it seems that exercise may stimulate the increase of Nix mRNA and, consequently, of mitophagy, although the mechanism by which exercise produces this increase is not clarified [88]. On the other hand, no changes are found in Nix mRNA in response to exercise in the muscle from aged subjects after a single bout of resistance training [68].

Bnip3 is another protein with no clear behavior in response to exercise. In fact, in two studies carried out in the skeletal muscle of active elderly without exercise intervention, opposite results were obtained (increase and decrease, resp.) [72, 87]. Moreover, no changes were found in Bnip3 mRNA after 24 weeks of combined exercise [89], or after a single bout of resistance training [68]. These contradictory responses could be due to methodological differences, because different exercise protocols were used.

Most of the researches carried out in the samples from human muscles seem to point that the level of physical activity does not change beclin 1 content in old subjects [72]. In the same line, beclin 1 remains unchanged in response to a single bout of resistance training [68] or to 8 weeks of strength training [75]. Nevertheless, an increase of mRNA beclin 1 level in the muscle from elderly individuals who practiced physical activity has been demonstrated [87]. The same results were obtained by our research group when analyzing the protein content in peripheral blood mononuclear cells, after 8 weeks of aerobic or resistance training $[2,10]$. On the other hand, LC3II or LC3II/I levels were increased in human muscle and mononuclear cells after a single bout of resistance training [68] or 8 weeks of aerobic or high intensity resistance training $[10,75]$. Likewise, a training program of 8 weeks of resistance training induced a nonsignificant increase in the LC3II/LC3I ratio in PBMCs from elderly participants [2]. Finally, brain levels of the autophagy marker LC3II were not significantly altered in the mouse after completing 3 weeks of treadmill running [83].

Joint analysis of all studies does not allow establishing a clear trend on the effect of physical activity in relation to the type of exercise, volume, or intensity. Overall, aerobic or combined chronic long-term training (more than 12 weeks) seems to be the type of exercise most effective to counteract the age-related damage at the mitochondrial level $[30,31,47,85,89]$. Therefore, it seems that exercise stimulates biogenesis, dynamics, and mitophagic capacity, although there is not a consensus about the behavior of different proteins in response to a physical stimulus. Future studies should focus on investigating the effects that different protocols of physical exercise can cause on the expression of mitochondrial proteins, as well as whether exercise practice could prevent mitochondrial damage and the effects on the dysfunctional mitochondria.

\section{Conclusions}

The maintenance of mitochondrial function and mitophagy is essential to the cells, since mitochondria are involved in both adaptive metabolism and survival in response to cellular stress. Although the studies analyzed do not exhibit a general consensus, it seems that aging impairs mitochondrial biogenesis and dynamics and decreases the mitophagic capacity of the organism. Several interventions, such as any type of physical exercise, are able to affect the activity and turnover of 
mitochondria by increasing biogenesis (specifically PGC- $1 \alpha$ and TFAM). In addition to, the changes detected in the biogenesis, aerobic, or combined long-term training also seem to produce increases in several markers of mitochondrial dynamics and mitophagy. However, we consider that it is very important to assess all these markers in different exercise protocols in order to establish a direct relationship between the detected changes and type, intensity, and volume of exercise. So, further research is necessary to determine the mechanisms of interaction between mitochondrial functions, aging, and physical activity, as well as to analyze possible factors that are supposed to influence these processes.

\section{Abbreviations}

Bcl-2: $\quad$ B-cell lymphoma 2 family

Bnip3: $\quad$ Adenovirus E1B $19 \mathrm{kDa}$-interacting protein 3

Bnip3L: Bnip3-like protein

Drp1: $\quad$ Dynamin-related protein 1

FUNDC1: FUN14 domain containing 1

GABARAP: Gamma-aminobutyric acid receptor-associated protein

GATE-16: Golgi-associated ATPase enhancer of $16 \mathrm{kDa}$

IMM: Inner mitochondrial membrane

LC3: $\quad$ Light chain 3

LIR: $\quad$ LC3-interacting region

Mfn: $\quad$ Mitofusin

MPTP: $\quad$ Mitochondrial permeability transition pore

mtDNA: $\quad$ Mitochondrial DNA

mtROS: $\quad$ Mitochondrial reactive oxygen species

mtUPR: Mitochondrial unfolded protein response

Nix: $\quad$ Nip3-like protein $\mathrm{X}$

NRF1: $\quad$ Nuclear respiratory factor 1

OMM: $\quad$ Outer mitochondrial membrane

OPA1: Optic atrophy protein 1

OXPHOS: Oxidative phosphorylation system

p62/ Sequestosome 1

SQSTM1:

Parkin: $\quad$ E3 ubiquitin protein ligase

PARL: Presenilin-associated rhomboid-like protein

PBMCs: $\quad$ Peripheral blood mononuclear cells

PGC-1 $\alpha$ : Receptor gamma coactivator 1-alpha

PINK1: $\quad$ PTEN-induced putative kinase 1

ROS: $\quad$ Reactive oxygen species

TFAM: $\quad$ Mitochondrial transcription factor A

Ubl: $\quad$ Ubiquitin-like

VDAC1: Voltage-dependent anion channel 1

$\Delta \Psi \mathrm{m}: \quad$ Mitochondrial membrane potential.

\section{Conflicts of Interest}

The authors declare that there is no conflict of interests regarding the publication of this paper.

\section{Acknowledgments}

This study was supported by Plan Nacional I+D+I DEP2013-47659-R, Spain. Brisamar Estébanez was supported by a fellowship from the Ministry of Education of Spain (FPU fellowship, Reference FPU15/05051). Osvaldo C. Moreira was supported by a fellowship from the Coordenação de Aperfeiçoamento de Pessoal de Nível Superior-CAPES/Brazil.

\section{References}

[1] C. López-Otín, M. A. Blasco, L. Partridge, M. Serrano, and G. Kroemer, "The hallmarks of aging," Cell, vol. 153, no. 6, pp. 1194-1217, 2013.

[2] Y. Mejías-Peña, B. Estébanez, P. Rodriguez-Miguelez et al., "Impact of resistance training on the autophagy-inflammationapoptosis crosstalk in elderly subjects," Aging (Albany, New York), vol. 9, no. 2, pp. 408-418, 2017.

[3] A. Schiavi and N. Ventura, "The interplay between mitochondria and autophagy and its role in the aging process," Experimental Gerontology, vol. 56, pp. 147-153, 2014.

[4] A. M. Cuervo and F. Macian, "Autophagy and the immune function in aging," Current Opinion in Immunology, vol. 29, pp. 97-104, 2014.

[5] K. Palikaras and N. Tavernarakis, "Mitophagy in neurodegeneration and aging," Frontiers in Genetics, vol. 3, p. 297, 2012.

[6] B. A. Payne and P. F. Chinnery, "Mitochondrial dysfunction in aging: much progress but many unresolved questions," Biochimica et Biophysica Acta (BBA) - Bioenergetics, vol. 1847, no. 11, pp. 1347-1353, 2015.

[7] M. S. Parihar and G. J. Brewer, "Simultaneous age-related depolarization of mitochondrial membrane potential and increased mitochondrial reactive oxygen species production correlate with age-related glutamate excitotoxicity in rat hippocampal neurons," Journal of Neuroscience Research, vol. 85, no. 5, pp. 1018-1032, 2007.

[8] S. P. Elmore, T. Qian, S. F. Grissom, and J. J. Lemasters, “The mitochondrial permeability transition initiates autophagy in rat hepatocytes," FASEB Journal, vol. 15, no. 12, pp. 22862287, 2001.

[9] K. Palikaras, E. Lionaki, and N. Tavernarakis, "Coupling mitogenesis and mitophagy for longevity," Autophagy, vol. 11, no. 8, pp. 1428-1430, 2015.

[10] Y. Mejías-Peña, P. Rodriguez-Miguelez, R. Fernandez-Gonzalo et al., "Effects of aerobic training on markers of autophagy in the elderly," Age (Dordrecht, Netherlands), vol. 38, no. 2, p. 33, 2016.

[11] G. López-Lluch, C. Santos-Ocaña, J. A. Sánchez-Alcázar et al., "Mitochondrial responsibility in ageing process: innocent, suspect or guilty," Biogerontology, vol. 16, no. 5, pp. 599620, 2015.

[12] A. Sanz, "Mitochondrial reactive oxygen species: do they extend or shorten animal lifespan?," Biochimica et Biophysica Acta (BBA) - Bioenergetics, vol. 1857, no. 8, pp. 1116-1126, 2016.

[13] I. Novak, "Mitophagy: a complex mechanism of mitochondrial removal," Antioxidants \& Redox Signaling, vol. 17, no. 5, pp. 794-802, 2012.

[14] H. N. Carter, C. C. Chen, and D. A. Hood, "Mitochondria, muscle health, and exercise with advancing age," Physiology, vol. 30, no. 3, pp. 208-223, 2015.

[15] K. Palikaras and N. Tavernarakis, "Mitochondrial homeostasis: the interplay between mitophagy and mitochondrial biogenesis," Experimental Gerontology, vol. 56, pp. 182-188, 2014.

[16] G. Gouspillou, N. Sgarioto, B. Norris et al., "The relationship between muscle fiber type-specific PGC-1 $\alpha$ content and 
mitochondrial content varies between rodent models and humans," PLoS One, vol. 9, no. 8, article e103044, 2014.

[17] P. Mishra and D. C. Chan, "Metabolic regulation of mitochondrial dynamics," The Journal of Cell Biology, vol. 212, no. 4, pp. 379-387, 2016.

[18] A. Hamacher-Brady and N. R. Brady, "Mitophagy programs: mechanisms and physiological implications of mitochondrial targeting by autophagy," Cellular and Molecular Life Sciences, vol. 73, no. 4, pp. 775-795, 2016.

[19] T. E. Kauppila, J. H. Kauppila, and N. G. Larsson, "Mammalian mitochondria and aging: an update," Cell Metabolism, vol. 25, no. 1, pp. 57-71, 2017.

[20] E. Schrepfer and L. Scorrano, "Mitofusins, from mitochondria to metabolism," Molecular Cell, vol. 61, no. 5, pp. 683-694, 2016.

[21] S. Hekimi, Y. Wang, and A. Noë, "Mitochondrial ROS and the effectors of the intrinsic apoptotic pathway in aging cells: the discerning killers!," Frontiers in Genetics, vol. 7, p. 161, 2016.

[22] N. Poulose and R. Raju, "Sirtuin regulation in aging and injury," Biochimica et Biophysica Acta (BBA) - Molecular Basis of Disease, vol. 1852, no. 11, pp. 2442-2455, 2015.

[23] M. B. Jensen and H. Jasper, "Mitochondrial proteostasis in the control of aging and longevity," Cell Metabolism, vol. 20, no. 2, pp. 214-225, 2014.

[24] A. M. Nargund, C. J. Fiorese, M. W. Pellegrino, P. Deng, and C. M. Haynes, "Mitochondrial and nuclear accumulation of the transcription factor ATFS-1 promotes OXPHOS recovery during the UPRmt," Molecular Cell, vol. 58, no. 1, pp. 123133, 2015.

[25] Y. Tian, C. Merkwirth, and A. Dillin, "Mitochondrial UPR: a double-edged sword," Trends in Cell Biology, vol. 26, no. 8, pp. 563-565, 2016.

[26] M. W. Pellegrino, A. M. Nargund, and C. M. Haynes, "Signaling the mitochondrial unfolded protein response," Biochimica et Biophysica Acta (BBA) - Molecular Cell Research, vol. 1833, no. 2, pp. 410-416, 2013.

[27] K. E. Conley, S. A. Jubrias, and P. C. Esselman, "Oxidative capacity and ageing in human muscle," Journal of Physiology, vol. 526, no. 1, pp. 203-210, 2000.

[28] D. L. Johannsen, K. E. Conley, S. Bajpeyi et al., "Ectopic lipid accumulation and reduced glucose tolerance in elderly adults are accompanied by altered skeletal muscle mitochondrial activity," The Journal of Clinical Endocrinology and Metabolism, vol. 97, no. 1, pp. 242-250, 2012.

[29] S. Larsen, M. Hey-Mogensen, R. Rabøl, N. Stride, J. W. Helge, and F. Dela, "The influence of age and aerobic fitness: effects on mitochondrial respiration in skeletal muscle," Acta Physiologica (Oxford), vol. 205, no. 3, pp. 423-432, 2012.

[30] A. R. Konopka, M. K. Suer, C. A. Wolff, and M. P. Harber, "Markers of human skeletal muscle mitochondrial biogenesis and quality control: effects of age and aerobic exercise training," The Journals of Gerontology. Series A, Biological Sciences and Medical Sciences, vol. 69, no. 4, pp. 371-378, 2014.

[31] N. T. Broskey, C. Greggio, A. Boss et al., "Skeletal muscle mitochondria in the elderly: effects of physical fitness and exercise training," The Journal of Clinical Endocrinology and Metabolism, vol. 99, no. 5, pp. 1852-1861, 2014.

[32] M. Z. Springer and K. F. Macleod, "Mitophagy: mechanisms and role in human disease," The Journal of Pathology, vol. 240, no. 3, pp. 253-255, 2016.
[33] W. X. Ding and X. M. Yin, "Mitophagy: mechanisms, pathophysiological roles, and analysis," Biological Chemistry, vol. 393, no. 7, pp. 547-564, 2012.

[34] A. Eiyama and K. Okamoto, "PINK1/Parkin-mediated mitophagy in mammalian cells," Current Opinion in Cell Biology, vol. 33, pp. 95-101, 2015.

[35] T. M. Durcan, M. Y. Tang, J. R. Pérusse et al., "USP8 regulates mitophagy by removing K6-linked ubiquitin conjugates from parkin," The EMBO Journal, vol. 33, no. 21, pp. 24732491, 2014.

[36] L. C. Gomes and L. Scorrano, "Mitochondrial morphology in mitophagy and macroautophagy," Biochimica et Biophysica Acta (BBA) - Molecular Cell Research, vol. 1833, no. 1, pp. 205-212, 2013.

[37] P. A. Ney, "Mitochondrial autophagy: origins, significance, and role of BNIP3 and NIX," Biochimica et Biophysica Acta (BBA) - Molecular Cell Research, vol. 1853, no. 10, Part B, pp. 2775-2783, 2015.

[38] N. Matsuda, "Phospho-ubiquitin: upending the PINK-Parkinubiquitin cascade," Journal of Biochemistry, vol. 159, no. 4, pp. 379-385, 2016.

[39] R. A. Gottlieb and R. S. Carreira, "Autophagy in health and disease. 5. Mitophagy as a way of life," American Journal of Physiology - Cell Physiology, vol. 299, no. 2, pp. C203-C210, 2010.

[40] K. E. Liu and W. A. Frazier, "Phosphorylation of the BNIP3 C-terminus inhibits mitochondrial damage and cell death without blocking autophagy," PLoS One, vol. 10, no. 6, article e0129667, 2015.

[41] C. Ploumi, I. Daskalaki, and N. Tavernarakis, "Mitochondrial biogenesis and clearance: a balancing act," FEBS Journal, vol. 284, no. 2, pp. 183-195, 2017.

[42] M. Gaugler, A. Brown, E. Merrell, M. DiSanto-Rose, J. A. Rathmacher, and T. H. Reynolds 4th, "PKB signaling and atrogene expression in skeletal muscle of aged mice," Journal of Applied Physiology, vol. 111, no. 1, pp. 192-199, 2011.

[43] A. M. Joseph, P. J. Adhihetty, N. R. Wawrzyniak et al., "Dysregulation of mitochondrial quality control processes contribute to sarcopenia in a mouse model of premature aging," PLoS One, vol. 8, no. 7, article e69327, 2013.

[44] M. F. O’Leary, A. Vainshtein, S. Iqbal, O. Ostojic, and D. A. Hood, "Adaptive plasticity of autophagic proteins to denervation in aging skeletal muscle," American Journal of Physiology Cell Physiology, vol. 304, no. 5, pp. C422-C430, 2013.

[45] R. A. Gioscia-Ryan, M. L. Battson, L. M. Cuevas, M. C. Zigler, A. L. Sindler, and D. R. Seals, "Voluntary aerobic exercise increases arterial resilience and mitochondrial health with aging in mice," Aging (Albany, New York), vol. 8, no. 11, pp. 2897-2914, 2016.

[46] A. M. Joseph, P. J. Adhihetty, T. W. Buford et al., "The impact of aging on mitochondrial function and biogenesis pathways in skeletal muscle of sedentary high- and low-functioning elderly individuals," Aging Cell, vol. 11, no. 5, pp. 801-809, 2012.

[47] C. Kang, E. Chung, G. Diffee, and L. L. Ji, "Exercise training attenuates aging-associated mitochondrial dysfunction in rat skeletal muscle: role of PGC-1 $\alpha$, Experimental Gerontology, vol. 48, no. 11, pp. 1343-1350, 2013.

[48] E. Koltai, N. Hart, A. W. Taylor et al., "Age-associated declines in mitochondrial biogenesis and protein quality control factors are minimized by exercise training," American Journal of Physiology - Regulatory, Integrative and Comparative Physiology, vol. 303, no. 2, pp. R127-R134, 2012. 
[49] R. Lanza, D. K. Short, K. R. Short et al., "Endurance exercise as a countermeasure for aging," Diabetes, vol. 57, no. 11, pp. 2933-2942, 2008.

[50] A. Safdar, M. J. Hamadeh, J. J. Kaczor, S. Raha, J. de Beer, and M. A. Tarnopolsky, "Aberrant mitochondrial homeostasis in the skeletal muscle of sedentary older adults," PLoS One, vol. 5, no. 5, article e10778, 2010.

[51] B. Chabi, V. Ljubicic, K. J. Menzies, J. H. Huang, A. Saleem, and D. A. Hood, "Mitochondrial function and apoptotic susceptibility in aging skeletal muscle," Aging Cell, vol. 7, no. 1, pp. 2-12, 2008.

[52] A. Picca, V. Pesce, F. Fracasso, A. M. Joseph, C. Leeuwenburgh, and A. M. S. Lezza, "Aging and calorie restriction oppositely affect mitochondrial biogenesis through TFAM binding at both origins of mitochondrial DNA replication in rat liver," PLoS One, vol. 8, no. 9, article e74644, 2013.

[53] J. Viña, M. C. Gomez-Cabrera, C. Borras et al., "Mitochondrial biogenesis in exercise and in ageing," Advanced Drug Delivery Reviews, vol. 61, no. 14, pp. 1369-1374, 2009.

[54] F. Derbré, M. C. Gomez-Cabrera, A. L. Nascimento et al., “Age associated low mitochondrial biogenesis may be explained by lack of response of PGC- $1 \alpha$ to exercise training," Age (Dordrecht, Netherlands), vol. 34, no. 3, pp. 669-679, 2012.

[55] M. M. Dinardo, C. Musicco, F. Fracasso et al., "Acetylation and level of mitochondrial transcription factor A in several organs of young and old rats," Biochemical and Biophysical Research Communications, vol. 301, no. 1, pp. 187-191, 2003.

[56] A. Picca, F. Fracasso, V. Pesce et al., "Age-and calorie restriction-related changes in rat brain mitochondrial DNA and TFAM binding," Age (Dordrecht, Netherlands), vol. 35, no. 5, pp. 1607-1620, 2013.

[57] A. Picca, V. Pesce, F. Fracasso, A. M. Joseph, C. Leeuwenburgh, and A. M. S. Lezza, "A comparison among the tissue-specific effects of aging and calorie restriction on TFAM amount and TFAM-binding activity to mtDNA in rat," Biochimica et Biophysica Acta (BBA) - General Subjects, vol. 1840, no. 7, pp. 2184-2191, 2014.

[58] Z. Bori, Z. Zhao, E. Koltai et al., "The effects of aging, physical training, and a single bout of exercise on mitochondrial protein expression in human skeletal muscle," Experimental Gerontology, vol. 47, no. 6, pp. 417-424, 2012.

[59] D. Capitanio, M. Vasso, S. De Palma et al., "Specific protein changes contribute to the differential muscle mass loss during ageing," Proteomics, vol. 16, no. 4, pp. 645-656, 2016.

[60] J. P. Leduc-Gaudet, M. Picard, F. St-Jean Pelletier et al., "Mitochondrial morphology is altered in atrophied skeletal muscle of aged mice," Oncotarget, vol. 6, no. 20, pp. 1792317937, 2015.

[61] K. L. Stauch, P. R. Purnell, and H. S. Fox, "Aging synaptic mitochondria exhibit dynamic proteomic changes while maintaining bioenergetic function," Aging (Albany, New York), vol. 6, no. 4, pp. 320-334, 2014.

[62] E. Bossy-Wetzel, M. J. Barsoum, A. Godzik, R. Schwarzenbacher, and S. A. Lipton, "Mitochondrial fission in apoptosis, neurodegeneration and aging," Current Opinion in Cell Biology, vol. 15, no. 6, pp. 706-716, 2003.

[63] D. Sebastián and A. Zorzano, "When MFN2 (mitofusin 2) met autophagy: a new age for old muscles," Autophagy, vol. 12, no. 11, pp. 2250-2251, 2016.

[64] D. Sebastián, E. Sorianello, J. Segalés et al., "Mfn2 deficiency links age-related sarcopenia and impaired autophagy to activation of an adaptive mitophagy pathway," The EMBO Journal, vol. 35, no. 15, pp. 1677-1693, 2016.

[65] J. Niemann, C. Johne, S. Schröder et al., “An mtDNA mutation accelerates liver aging by interfering with the ROS response and mitochondrial life cycle," Free Radical Biology \& Medicine, vol. 102, pp. 174-187, 2017.

[66] G. Distefano, R. A. Standley, J. J. Dubé et al., "Chronological age does not influence ex-vivo mitochondrial respiration and quality control in skeletal muscle," The Journals of Gerontology Series A: Biological Sciences and Medical Sciences, vol. 72, no. 4, pp. 535-542, 2017.

[67] D. Capitanio, R. Leone, C. Fania, E. Torretta, and C. Gelfi, "Sprague Dawley rats: a model of successful heart aging," EuPA Open Proteomics, vol. 12, pp. 22-30, 2016.

[68] D. I. Ogborn, B. R. McKay, J. D. Crane et al., "Effects of age and unaccustomed resistance exercise on mitochondrial transcript and protein abundance in skeletal muscle of men," American Journal of Physiology - Regulatory, Integrative and Comparative Physiology, vol. 308, no. 8, pp. R734R741, 2015.

[69] L. A. Scarffe, D. A. Stevens, V. L. Dawson, and T. M. Dawson, "Parkin and PINK1: much more than mitophagy," Trends in Neurosciences, vol. 37, no. 6, pp. 315-324, 2014.

[70] O. Krestinina, T. Azarashvili, Y. Baburina et al., "In aging, the vulnerability of rat brain mitochondria is enhanced due to reduced level of $2^{\prime}, 3^{\prime}$-cyclic nucleotide-3'-phosphodiesterase (CNP) and subsequently increased permeability transition in brain mitochondria in old animals," Neurochemistry International, vol. 80, pp. 41-50, 2015.

[71] M. Gram, A. Vigelsø, T. Yokota et al., "Two weeks of one-leg immobilization decreases skeletal muscle respiratory capacity equally in young and elderly men," Experimental Gerontology, vol. 58, pp. 269-278, 2014.

[72] S. Zampieri, L. Pietrangelo, S. Loefler et al., "Lifelong physical exercise delays age-associated skeletal muscle decline," The Journals of Gerontology Series A: Biological Sciences and Medical Sciences, vol. 70, no. 2, pp. 163-173, 2015.

[73] F. Ko, P. Abadir, R. Marx et al., "Impaired mitochondrial degradation by autophagy in the skeletal muscle of the aged female interleukin 10 null mouse," Experimental Gerontology, vol. 73, pp. 23-27, 2016.

[74] G. Sgarbi, P. Matarrese, M. Pinti et al., "Mitochondria hyperfusion and elevated autophagic activity are key mechanisms for cellular bioenergetic preservation in centenarians," Aging (Albany, New York), vol. 6, no. 4, pp. 296-310, 2014.

[75] R. E. Tanner, L. B. Brunker, J. Agergaard et al., "Age-related differences in lean mass, protein synthesis and skeletal muscle markers of proteolysis after bed rest and exercise rehabilitation," The Journal of Physiology, vol. 593, no. 18, pp. 42594273, 2015.

[76] B. K. Pedersen and B. Saltin, "Exercise as medicine - evidence for prescribing exercise as therapy in 26 different chronic diseases," Scandinavian Journal of Medicine \& Science in Sports, vol. 25, pp. 1-72, 2015.

[77] B. T. Tam and P. M. Siu, "Autophagic cellular responses to physical exercise in skeletal muscle," Sports Medicine, vol. 44, no. 5, pp. 625-640, 2014.

[78] E. Barbieri, D. Agostini, E. Polidori et al., "The pleiotropic effect of physical exercise on mitochondrial dynamics in aging skeletal muscle," Oxidative Medicine and Cellular Longevity, vol. 2015, Article ID 917085, 15 pages, 2015. 
[79] O. M. Palacios, J. J. Carmona, S. Michan et al., "Diet and exercise signals regulate SIRT3 and activate AMPK and PGC- $1 \alpha$ in skeletal muscle," Aging (Albany, New York), vol. 1, no. 9, pp. 771-783, 2009.

[80] V. Ljubicic, A. M. Joseph, P. J. Adhihetty et al., "Molecular basis for an attenuated mitochondrial adaptive plasticity in aged skeletal muscle," Aging (Albany, New York), vol. 1, no. 9, pp. 818-830, 2009.

[81] J. N. Cobley, J. D. Bartlett, A. Kayani et al., "PGC-1a transcriptional response and mitochondrial adaptation to acute exercise is maintained in skeletal muscle of sedentary elderly males," Biogerontology, vol. 13, no. 6, pp. 621-631, 2012.

[82] N. Iversen, P. Krustrup, H. N. Rasmussen, U. F. Rasmussen, B. Saltin, and H. Pilegaard, "Mitochondrial biogenesis and angiogenesis in skeletal muscle of the elderly," Experimental Gerontology, vol. 46, no. 8, pp. 670-678, 2011.

[83] A. M. Gusdon, J. Callio, G. DiStefano et al., "Exercise increases mitochondrial complex I activity and DRP1 expression in the brains of aged mice," Experimental Gerontology, vol. 90, pp. 1-13, 2017.

[84] M. Picard, B. J. Gentil, M. J. McManus et al., "Acute exercise remodels mitochondrial membrane interactions in mouse skeletal muscle," Journal of Applied Physiology, vol. 115, no. 10, pp. 1562-1571, 2013.

[85] C. E. Fealy, A. Mulya, N. Lai, and J. P. Kirwan, "Exercise training decreases activation of the mitochondrial fission protein dynamin-related protein-1 in insulin-resistant human skeletal muscle," Journal of Applied Physiology, vol. 117, no. 3, pp. 239245, 2014.

[86] J. S. Ju, S. I. Jeon, J. Y. Park et al., "Autophagy plays a role in skeletal muscle mitochondrial biogenesis in an endurance exercise-trained condition," The Journal of Physiological Sciences, vol. 66, no. 5, pp. 417-430, 2016.

[87] M. J. Drummond, O. Addison, L. Brunker et al., "Downregulation of E3 ubiquitin ligases and mitophagy-related genes in skeletal muscle of physically inactive, frail older women: a cross-sectional comparison," The Journals of Gerontology Series A: Biological Sciences and Medical Sciences, vol. 69, no. 8, pp. 1040-1048, 2014.

[88] C. Jamart, N. Benoit, J. M. Raymackers, H. J. Kim, C. K. Kim, and M. Francaux, "Autophagy-related and autophagyregulatory genes are induced in human muscle after ultraendurance exercise," European Journal of Applied Physiology, vol. 112, no. 8, pp. 3173-3177, 2012.

[89] S. E. Wohlgemuth, H. A. Lees, E. Marzetti et al., "An exploratory analysis of the effects of a weight loss plus exercise program on cellular quality control mechanisms in older overweight women," Rejuvenation Research, vol. 14, no. 3, pp. 315-324, 2011. 


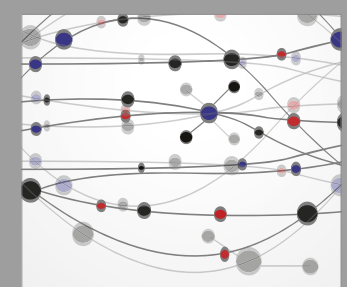

The Scientific World Journal
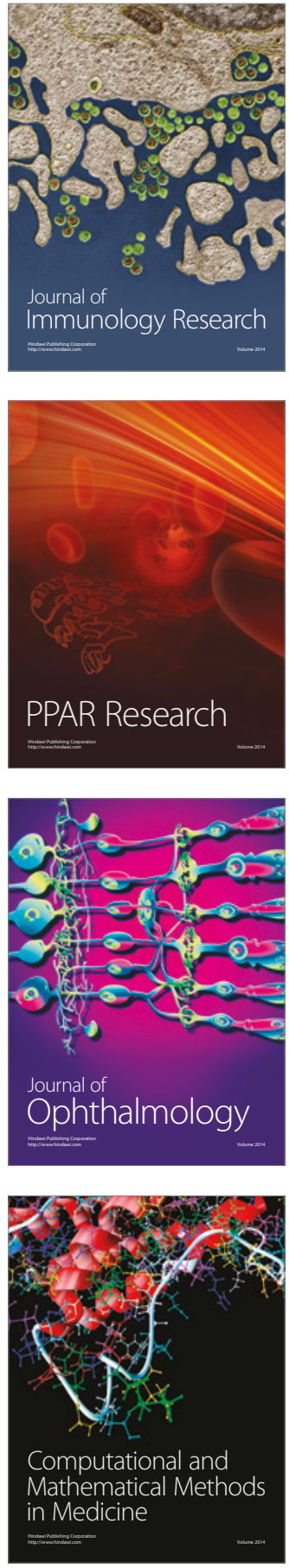

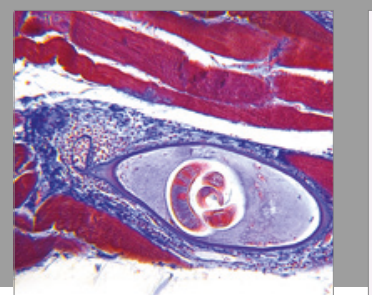

Gastroenterology Research and Practice
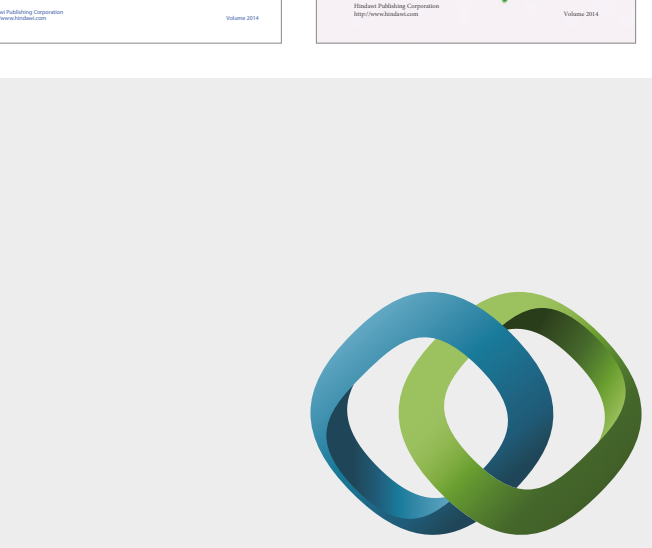

\section{Hindawi}

Submit your manuscripts at

https://www.hindawi.com
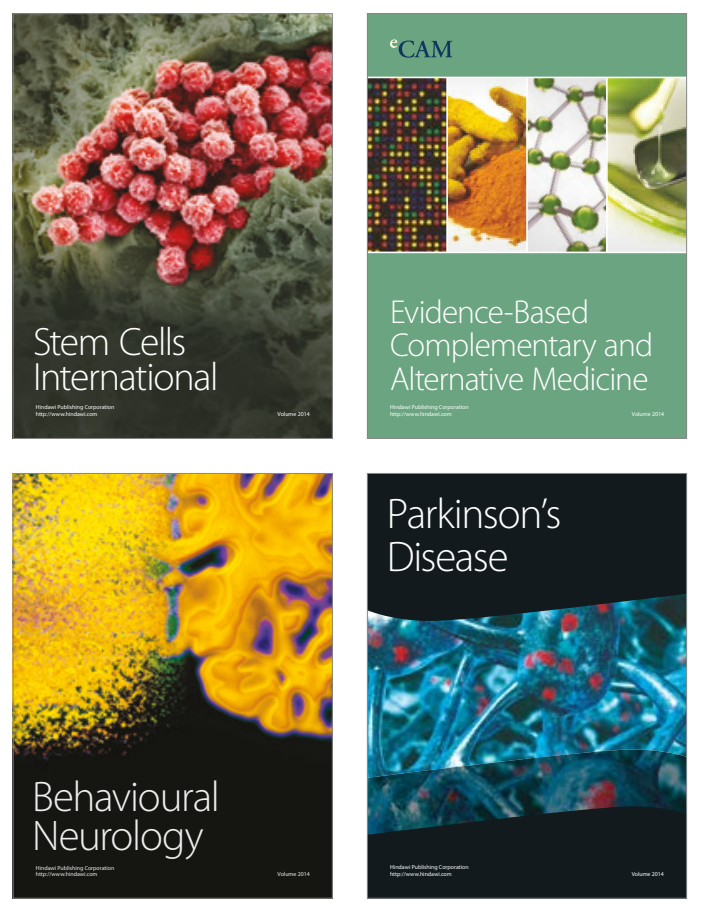
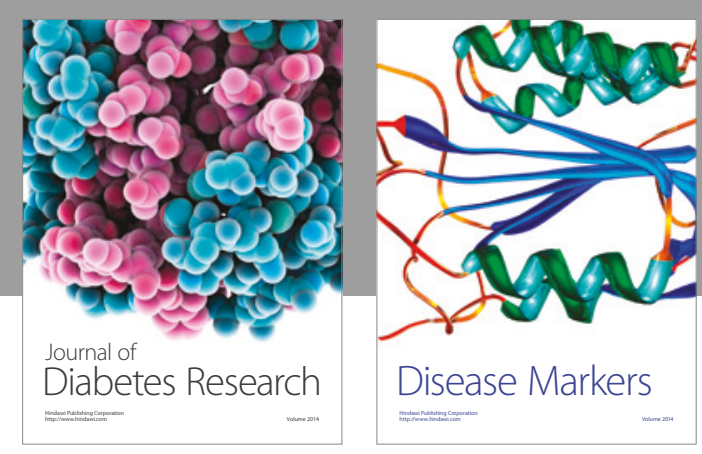

Disease Markers
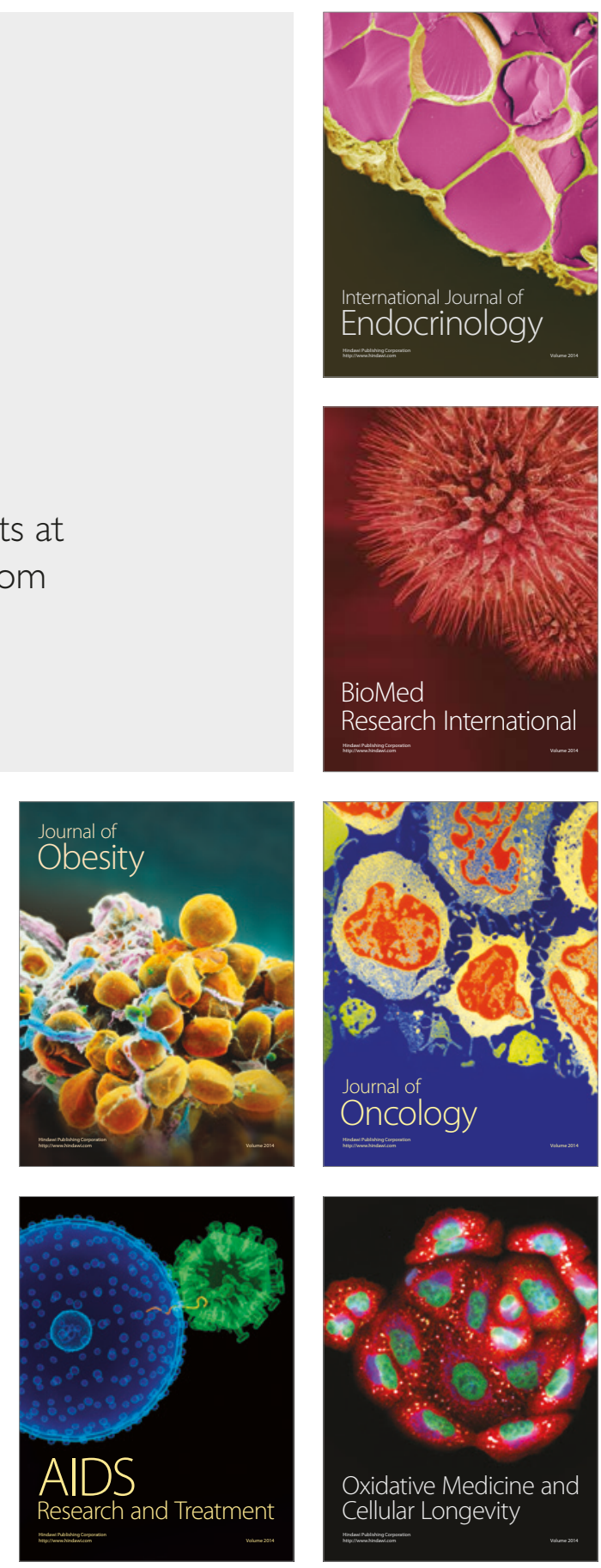